\title{
Research on Development Paths of Sichuan Pepper Industry under the Background of Rural Revitalization Strategy
}

\author{
Yu He${ }^{1}$, Wenkuan Chen ${ }^{1, *}$ \\ ${ }^{1}$ Sichuan Agricultural University, Chengdu, 611130, China \\ *Corresponding Author: Wenkuan Chen
}

Keywords: Sichuan pepper, Industry development, Industry convergence, Industry revitalization

\begin{abstract}
Under the background of rural revitalization, the impetus for the development of Sichuan pepper industry needs further improvement. Combining with the needs of modern agricultural development and rural revitalization strategy, this paper focuses on improving the quality and efficiency of Sichuan pepper, promoting the sustainable development of Sichuan pepper industry. The development paths of Sichuan pepper Industry include implementing Sichuan pepper industry integration development, improving the quality of Sichuan pepper industry, building Sichuan pepper industry risk prevention, expanding the investment and financing channels of Sichuan pepper industry and cultivating Sichuan pepper industry revitalization talent.
\end{abstract}

In the Opinions on Implementing the Strategy of Rural Revitalization issued by the State Council of the Central Committee of the Communist Party of China in 2018, it is pointed out that rural revitalization and industrial prosperity are the key points, and the quality of agricultural development must be improved to cultivate new momentum for rural development. Sichuan pepper (Sichuan pepper, pepper) has a long history, has been an important channel for Sichuan Province to develop the rural economy and increase farmers' income, in recent years it has become a major industry choice for poverty alleviation. How to solve the contradiction between production and marketing, enhance market competitiveness and industrial influence, and continuously improve the income of industrial population, especially farmers in poor areas, is a major issue for the future development of Sichuan pepper industry. Strengthening Sichuan pepper industry has become the practical need to implement the strategy of Rural Revitalization and promote the development of rural economy in Sichuan province.

\section{Promote Convergence Development of Sichuan Pepper Industry}

\subsection{Optimize the Industrial Structure of the Dominant Producing Areas, and Accelerate the Development of Sichuan Pepper Producing Areas}

First, the raw material supply of Sichuan pepper should be stabilized. The quality and quantity of raw material supply should be stabilized by the long-term cooperation of the processing subject. Therefore, modern technology management and service system should be used and established from the raw material links to keep the quality and quantity of Sichuan pepper production relatively stable, and standardize picking to change the roughness. The extraction of Sichuan style pepper lays the foundation for initial processing and intensive processing. On this basis, the primary processing of Sichuan pepper was developed in the main suitable areas of Sichuan Province, relying on the advantages of enterprises. Establish the whole industry chain standards to ensure that the quality of the initial processing of Sichuan pepper can meet the needs of all kinds of main bodies, integrate traditional technology and modern science and technology, form a unique regional primary processing technology and technology, improve the quality and quantity of the initial processing of Sichuan pepper, give full play to the spices, food and other aspects of daily cuisine. 


\subsection{Establish Sichuan Pepper Industry Alliance}

The industrial alliance should play an important role in the development of Sichuan pepper industry in various industrial links, industrial chain, and strive to build an information technology service platform, providing supply and marketing, planting and other links as one of the information collections. Actively submit proposals to the relevant departments to apply for expert research on the development of Sichuan pepper industry. Attention should be paid to the external study and exchange of industrial alliances, to the study of foreign advanced planting and processing technologies, and to the development of Sichuan pepper in accordance with local conditions. Establish a social service team to provide technical services to Sichuan pepper harvesting, pest control, UAV application, mechanization or semi-mechanization, solve the problem of labor shortage, standardize the development of Sichuan pepper industry, improve the competitiveness of Sichuan pepper industry, and promote the quality and efficiency of Sichuan pepper industry.

\subsection{Explore the Management Mode of "Platform + Base + Farmer"}

Introduce powerful technology enterprises, establishing Sichuan pepper high value-added products e-commerce platform, establishing a sound management system, including logistics transport monitoring, monitoring strict storage management equipment. Sichuan pepper enterprises provide food planting standards and agricultural production, farmers are responsible for planting management, Sichuan pepper alliance platform and various colleges and universities to provide technical personnel to guide planting, the platform records all data, to ensure transparency of the planting end. In the processing and circulation links, establish a platform to cooperate with Sichuan pepper processing enterprises, warehousing and logistics companies, establish a traceable, cheating-proof, traceable, monitorable certification management system, a separate closed pipeline management mode, remote real-time monitoring of Internet of Things equipment, consumers can track each commodity in real time.

\section{Promote Quality Enhancement of Sichuan Pepper Industry}

\subsection{Appropriate Development of Sichuan Pepper Scale}

Changing the development thinking of Sichuan pepper, reasonably controlling the planting scale of Sichuan pepper, and controlling the behavior of occupying farmland land, public welfare forest and other economic forest land because of blindly expanding the planting scale and expanding the industrial scale. Promote Sichuan pepper industry to improve quality and efficiency. Based on the moderate increase of the quantity and scale of Sichuan pepper, Sichuan pepper culture and food should be promoted to the world. The popularity of Sichuan pepper products should be aroused, the supply of Sichuan pepper to domestic and foreign products should be improved, and the management benefit of Sichuan pepper should be improved.

\subsection{Implement High-quality Native Varieties Breeding and Introduction Projects}

The implementation of the improved breeding system of Sichuan pepper based on local fine varieties is promoted. Promote joint breeding between scientific research institutes and enterprises to tackle key problems, strengthen resistance breeding of Sichuan pepper to conventional diseases, develop and optimize several high-yielding and stress-resistant varieties suitable for different climatic environments in our province, strengthen the promotion of new varieties already developed, and achieve centralized breeding and unified seedling supply. We should carry out the purification and rejuvenation of Sichuan pepper varieties, standardize the traditional seedling cultivation methods, reduce the confounding situation of Sichuan pepper, and keep the original fine characters of Sichuan pepper by seed propagation, ramet, cutting and grafting to speed up the popularization and application of fine varieties. We should develop professional seedling-raising subjects, encourage the development of seedling-raising and breeding subjects, establish scientific and technological incubation bases for Sichuan pepper, standardize seedling-raising and breeding links from scientific 
and technological breeding and seedling-raising, and provide high-quality varieties for Sichuan pepper industry.

\subsection{Strengthen the Quality Safety Supervision of Sichuan Pepper Agricultural Products}

Strengthen the environmental monitoring of agricultural origin, unify the periodic sampling inspection of Sichuan pepper brand agricultural products, strengthen the export of agricultural product quality and safety risk detection, establish and improve the monitoring results notification system and quality integrity system. We will intensify administrative law enforcement and supervision over agricultural inputs, and severely investigate and punish illegal acts such as the production, sale, use and illegal addition of prohibited pesticides. By using "block chain" technology, the quality traceability management at provincial, municipal, county and township levels will be strengthened, and a quality traceability management platform for brand agricultural products will be established. The quality traceability system of the Internet of Things will be established by leading brand agricultural products enterprises to use electronic information technology to realize the storage of production records, the traceability of product flow and the inquiry of storage and transportation information. To implement the punitive damages system of commodity quality, we should prohibit illegal enterprises and responsible persons from entering the market in accordance with the law.

\section{Construct Risk Prevention Mechanism of Sichuan Pepper Industry}

\subsection{Perfect the Service System of Science and Technology and Improve Application Level of Science and Technology in Disaster Prevention}

To ensure the timeliness of disaster prevention and control, it is necessary to establish a comprehensive pre-disaster monitoring and early warning system in Sichuan pepper industry development areas, and to associate the occurrence of natural disasters with agricultural pest control and forest fire prevention. Adopt engineering technology and ecological maintenance measures, use scientific agricultural irrigation technology, cultivate excellent varieties of Sichuan pepper, improve the resistance of Sichuan pepper varieties to natural drought and pest resistance. Establish a scientific and technological service platform for Sichuan pepper industry, provide various kinds of technical support, establish accurate scientific and technological service channels, provide various kinds of technical guidance and management guidance for farmers, improve the management level of the main business.

\subsection{Establish an Effective Decision-Making System}

Establishing an effective decision-making system is to form a system of information sharing, coordination, effectiveness and standardization of management among the main business entities and industrial participants. In the industrialized management mode of "company + farmer", "cooperative economic organization + farmer" and "specialized market + farmer", it is necessary to establish a standardized corporate governance structure and make clear the interests among shareholders, directors, supervisors and managers. Give full play to the advantages of the main body of the company, cooperative economic organizations and other subjects, pay attention to the training of the main business, including its theoretical knowledge level, technical application level and management system level of training, by the government-led, cultivate Sichuan pepper industry professional managers. Encourage the establishment of agricultural risk consulting companies, the company for Sichuan pepper industry information sharing, risk assessment, information consultation and publication. To reduce decision-making errors, do well in information dissemination and protect farmers' interests.

\subsection{Develop Cooperative Insurance}

To explore the establishment of cooperative insurance organizations with the aim of mutual aid and mutual benefit, without seeking profit, and with the aim of reducing costs, can solve the problem 
that the general insurance companies are unwilling to operate agricultural insurance. Supports the establishment of regional cooperative insurance, the establishment of insurance cooperatives, the establishment of insurance cooperative alliances, insurance cooperatives, if there is a surplus in the current year premium, in principle, should be retained as a reserve, without affecting the payment of the case, to participate in financial circulation, such as losses, in the co-operatives between adjustments, if unable to adjust, to The national insurance company applies for compensatory adjustment to ensure its stability and continuity.

\section{Broaden the Trench of Investment and Financing of Sichuan Pepper Industry}

\subsection{Strengthen Financial Support to Realize the Breeding and Technology Development of Sichuan Pepper}

Increase financial input, fully introducing scientific research personnel, equipment and popularization resources both inside and outside the province, forming technical teams, fully introducing standardized production and processing technology and intelligence of developed countries, taking "quality assurance, yield enhancement and efficiency enhancement" as the core, speeding up the breeding and popularization of high-quality, high-yield and multi-resistant hybrid varieties with local characteristics, and The research and popularization of fast, light, simplified and ecological cultivation techniques will be strengthened. The research and application of special facilities and equipment, agricultural machinery and special fertilizers will be strengthened. The industrialization of traditional processing products will be accelerated and the development of new products with diversified and high added value will be accelerated. We will strengthen the introduction, comparison test, purification and rejuvenation, supporting cultivation technology test and technical training, and carry out technical application and industrial development model demonstration, certification, brand building and market development.

\subsection{Speed Up the Construction of Service System}

Taking each enterprise (cooperative) and its driving farmers as project units, according to the enterprise (cooperative) + base + farmer model, the construction regulations take each enterprise (cooperative) and its driving farmers as project units, and according to the enterprise (cooperative) + base + farmer model, the large-scale production base is built, and the ecological cultivation techniques are demonstrated. We will improve green prevention and control facilities, the Internet of Things, e-commerce, quality traceability system and other standard garden construction facilities (equipment); and build a complete set of intensive seedling-raising centers, machine-cultivated roads, production conveniences, irrigation and drainage ditches, reservoirs, water conservancy pipe networks and other field infrastructure to support leading enterprises, carry out brand building, publicity and promotion. Regional market and market construction of producing areas. At the same time, we should increase financial input in market construction. Relying on the Sichuan pepper specialized market, according to the idea of online and offline collaborative promotion, we should strengthen the construction of the market information service system of Sichuan pepper industry, vigorously develop e-commerce with enterprises as the main body, introduce the Sichuan pepper Specialized Committee of the National Vegetable Circulation Association, and enhance the market influence, popularity and reputation of Sichuan pepper in northern Guizhou.

\subsection{Give Full Play to the Advantages of Free Trade Pilot Area Opening to the Outside World}

We should strengthen the linkage between financial innovation in free trade pilot area and the construction of financial centers in the west, and explore the establishment of a cross border financial service center focusing on the area along the "one belt and one road" area. We will deepen the centralized operation and management of foreign exchange funds at the headquarters of multinational corporations and innovate two-way RMB fund pools, strive for pilot reform in the management of foreign debt slices, and facilitate cross-border investment and financing of enterprises. We will 
support the development of new financing modes such as commercial factoring and financial leasing, and explore regulatory and wind control modes suitable for the development of the new financial industry. Promote the development of agricultural industry with Sichuan pepper as the main product.

\section{Cultivate Qualified Talents of Sichuan Pepper Industry}

\subsection{Pay Attention to the Team Building of Sichuan Pepper Entrepreneurs}

To play the role of entrepreneurs, in addition to the "two hands" of the government and the market, we should also create a sustained and healthy "growth environment", that is, the rule of law environment, market environment, social environment, the focus of the rule of law environment. The key is to protect the legitimate rights and interests of entrepreneurs according to law, ensure fair competition and honest management, and respect and encourage entrepreneurship. Accelerate the transformation and application of scientific and technological achievements and create an online and offline platform for the transformation of scientific and technological achievements. Hand in hand with the provincial academy of agricultural sciences and Sichuan pepper industrialization leading enterprises to establish a joint technology innovation center, and enterprises jointly declare, undertake national science and technology innovation projects, in the implementation of the project use and training personnel. To organize and carry out industry personnel training activities, focusing on the training of scientific and technological innovation and promotion personnel, business management personnel, entrepreneurs, and professional skills, to promote and guide local organizations to carry out extensive personnel training, and to introduce several outstanding rural entrepreneurs and rural craftsmen of Sichuan pepper.

\subsection{Grasp Foundation, Strengthen Training, Improve Professional Level of Sichuan Pepper Technical Personnel and Strive to Achieve Self-Cultivation of Talents}

With the combination of training, support and incentives, the graduates of agriculture-related specialties should be guided to work in Grass-roots Agricultural Technology Extension Service institutions. Establish a long-term training mechanism, adopt visiting and learning, centralized classes, on-site training, network teaching and other ways, strengthen grass-roots agricultural technical personnel knowledge and skills training, train several theoretical and technical Sichuan pepper local experts. Agricultural technical personnel training should resolutely implement the strategic spirit of invigorating agriculture through science and education and strengthening agriculture by talents. In accordance with the needs of cultivating "one understanding and two love" Sichuan pepper rural talents, the proportion of on-site teaching and case teaching should be further increased in the selection of teaching content, and duplicative and operable cases should be selected to enable students to master more advanced cases.

\subsection{Innovate Mechanism and Take Measures to Enhance the Effectiveness of Nurturing New Professional Farmers}

We will improve the "one master, multiple" education and training system for new-type vocational farmers, and make overall use of public welfare training resources such as agricultural and broadcasting schools, agricultural-related colleges, agricultural research institutes, agricultural technology popularization institutions, etc. to foster new-type vocational farmers. We should give full play to the role of market mechanism, encourage and support qualified Sichuan pepper enterprises and other market players to participate in the cultivation work through government purchasing services and market-oriented operation, and promote the cultivation of new professional farmers to face industry, integrate into industry and service industry. We should deepen the integration of production and education, school-enterprise cooperation, give full play to the role of agricultural vocational education groups, support local integration of resources to run and broaden the channels for cultivating new-type professional farmers. Sichuan Pepper Park and enterprises should be encouraged to give full play to their own advantages, establish new vocational practice and 
training bases and start-up incubation bases, guide peasant cooperatives to establish peasant field schools, and provide new professional peasants with learning, teaching observation, practice and start-up incubation sites.

\section{References}

[1] Zhang Qian. Cultural Interpretation of Sichuan People Eating Pepper Food [J]. China Condiment, 2016, 41(10): 128-132.

[2] Ri Li Weiri, Yang Defu. Developing Forestry Industry Is Impor tant Countermeasures for Economy Revitalization in Minor ity Areas - The Investigation ofDevelopingGreen Pepper Industry of JingyangCounty in Sichuan [J]. China Forestry Economy, 2011(4): 21-25.

[3] Huang Renzhong, Huang Qizhong, Zhang Shicai, et al. Present Situation and Developmental Proposals of Processing Type Pepper Industry in Chongqing [J]. Journal of China Capsicum, 2018(1): 4-7.

[4] Hou Guanxiang. Introduction of Pepper to China and Formation of the Sichuan and Hunan Culinary Families [J]. Journal of Chuxiong Normal Unversity, 2018, 33(2): 1-12. 